\title{
A Field Programmable Gate Array (FPGA) Based Non-Linear Filters for Gas Turbine Prognostics
}

\author{
Jayant Kumar Nayak $^{1}$, Vatsala Prasad ${ }^{1}$, and Ranjan Ganguli ${ }^{2}$ \\ ${ }^{1}$ Electrical and Electronics Engineering, Manipal Institute of Technology, Manipal, India \\ jayant31121997@gmail.com,vatsala.prasad1998@gmail.com \\ ${ }^{2}$ Professor, Aerospace Engineering, Indian Institute of Science, Bangalore, India \\ ganguli@iisc.ac.in
}

\begin{abstract}
The removal of noise from signals obtained through the health monitoring systems in gas turbines is an important consideration for accurate prognostics. Several filters have been designed and tested for this purpose, and their performance analysis has been conducted. Linear filters are inefficient in the removal of outliers and noise because they cause smoothening of the sharp features in the signal which can indicate the onset of a fault event. On the other hand, nonlinear filters based on image processing methods can provide more precise results for gas turbine health signals. Among others, the weighted recursive median (WRM) filter has been shown to provide greater accuracy due to its weight adaptability depending on the signal type. However, sampling data at high rates is possible which needs hardware implementation of the filter. In this paper, the design, simulation and implementation of WRM filters on the FPGA (Field Programmable Gate Arrays) platforms Vivado Design Suite by Xilinx and Quartus Pro Lite Edition 19.3 has been performed. The architectural detail and performance result with the FPGA filters when subjected to abrupt and gradual fault signal is presented.
\end{abstract}

\section{INTRODUCTION}

A gas turbine engine forms the core propulsion system of most aircraft, supplying it with power to maintain its flight. Therefore, it becomes essential to undertake steps so as to ensure their healthy working condition. Over the course of its constant usage and because of the strenuous conditions under which it operates, the engine may undergo faults. These faults include foreign/domestic object damage, corrosion, erosion and/or fouling. Health monitoring methods to track and prevent these faults, predict remaining life and suggest future

Jayant Kumar Nayak et al. This is an open-article distributed under the terms of the Creative Commons Attribution 3.0 United States License, which permits unrestricted use, distribution, and reproduction in any medium, provided the original author and source are credited.

https://doi.org/10.36001/IJPHM.2021.v12i3.2960 actions has become a widely researched domain, and the technology is constantly developing (Volponi, 2014; Borguet, Henriksson, McKelvey and Léonard, 2011). Several investigations have been carried out to review the developments in this area (Zhao, Wen and Li, 2016; Fentaye, Baheta, Gilani and Kyprianidis, 2019).

The basic parameters that are used to determine the health of engines, namely, exhaust gas temperature (EGT), low rotor speed (N1), high rotor speed (N2), and fuel flow (WF), are susceptible to deviations and noise contaminations, due to a variety of factors which may involve channel noise or measurement errors at the source itself. Methods to check and inhibit the noise have inspired numerous researchers, which can be broadly classified under filtering, neural networks, least-square estimation and probabilistic approaches. High quality measurement data is the key to accurate prognostics.

The use of linear filters, such as the FIR (finite impulse response) filter in smoothing out deviations of data from a baseline case caused due to the existence of noise, their advantages and disadvantages has been discussed in detail in by Ganguli (2012). Ganguli (2012) demonstrated the superiority of non-linear filters such as median filter in removing the outliers in gas path measurement data, and successfully simulated a non-linear idempotent median filter which preserved the sharp changes in the signal which herald a fault event. The Kalman filter, which determines an estimate of unknowns whose measurements are taken over time, also finds widespread use in gas turbine prognostics. A Kalman filter-based algorithm for the estimation of engine performance with proof pertaining to flight simulation and actual data was presented (Luppold, Roman, Gallops and Kerr, 1989). This finds application in flight maintenance and aircraft performance diagnostics. Pourbabaee, Meskin and Khorasani (2016) proposed a multiple-model scheme based on a hybrid Kalman filter to track fault detection, isolation and identification (FDII), and compared the soundness of their model over various other multiple model schemes which make use of Kalman filters. A hybrid Kalman filter 
architecture consisting of linearized KF (LKF) and Extended $\mathrm{KF}(\mathrm{EKF})$, master filters and federated filtering structure was demonstrated and its robustness in sensing faults was established by Lu, HuangY., Huang J., and Qiu (2018). Its performance was noted to be better than other centralized KFs, due to the combination of linear and non-linear KFs which provides an optimum state estimate. In another work, $\mathrm{Lu}$ et al developed a novel Extended Kalman Filter (EKF) algorithm approach for estimation of the deterministic state of gas turbine engines (Lu, Ju and Huang, 2016).

A hybrid structure was proposed which consisted of the particle filtering technique to determine the state of the parameters which define the health of the system (Daroogheh, Baniamerian, Meskin and Khorasani, 2015). The observations were fed into an artificial neural network that provides predictions for future state of the parameters when the cause of damage to the turbine is known. The study was conducted on performance degradation caused by fouling and the resultant hybrid network proved to be better than typical neural networks. Another feasible technique for fault detection in the Exhaust Gas Temperature (EGT) is based on applying a convolutional neural network to obtain the value difference between two consecutive observations in EGT profile, which improves the sensitivity of anomaly detection (Liu, Liu, Yu, Kang, Yan, Wang, and Pecht, 2018). A methodology based on dynamic neural network has been verified and its advantages have been studied in recognizing faults in a turbofan engine (Tayarani-Bathaie, Vanini, and Khorasani, 2012).

Several genetic algorithm-based methods have been suggested for the improvement in the accuracy of prediction in faults of gas turbine engines. For example, a genetic algorithm based multi-point performance technique has been studied, which carries out calibration of the simulated data and the actual engine performance at different off-design settings, wherein the algorithm is employed to find optimum scaling factor functions (Li, Ghafir, Wang, Singh, Huang and Feng, 2011). The algorithm was tested on a single spool turboshaft gas turbine and showed significant improvement in performance. However, its credibility is challenged by drawbacks such as excessive time consumption and search for an appropriate range of scaling factor coefficients to obtain optimum scaling factor functions. To overcome this, another method based on the least square method has been proposed, which provides a more deterministic alternative to find this range (Li, Ghafir, Wang, Singh, Huang and Feng, 2012).

All prognostic methods depend on high quality data for their successful performance. However, filters and other algorithms used for prognostics can be expensive in terms of computation time and may not have real time capability. A hardware implementation of the filters used for gas turbine prognostics is therefore attractive. The growth of FPGA (Field Programmable Gated Array) usage in the consumer domain has seen a rapid increase over the past years, making its way into a variety of industrial applications such as that of automation, computation, networking and communication, aerospace etc. Particularly in communication, the wireless sensor networks deployed for radio interfaces for aerospace systems has been explored, by means of prototyping a Single Carrier Frequency Division Multiple Access (SC-FDMA) on models such as the Virtex 2,4,5 and Stratix 3 (Henaut, Dragomirescu and Plana, 2009). FPGAs provide performance that is comparable to that of custom-made chips at a reasonable cost and proves to be a more viable alternative. However, FPGA are prone to soft errors (Fay, Campbell, Miller and Connors, 2007) that due to which their usage in the aerospace industry has been limited. But with technological advancement in the design techniques of FPGAs their potential is being progressively exploited. The autonomous systems that are deployed on rovers for planetary exploration require the capability to learn so as to deal with unexpected circumstances when in flight. For this purpose as well, the implementation of neural network on FPGA has been studied (Gankidi and Thangavelautham, 2017). In the domain of aircraft, the use of filters for fault detection in gas turbines is being researched consistently. It is of interest, thus, to view the performance of these filters when implemented on FPGAs, so as to verify their potential use in the measurement of deviation from ideal behaviour of gas turbine engines that serves the purpose of health monitoring. This prognostics filter problem is useful to further the spread of FPGA technology in the prognostics community. This paper presents the simulation results of the averaging, median, recursive median as well as the weighted recursive median on the Xilinx Vivado Design Suite as well as the Intel Quartus Development Suite and presents the results of the comparative study performed when test data is fed into these filters.

Another commonly used processor is the GPU which stands for Graphical Processing Unit. GPUs and FPGA are popular competitors for various applications such as robotics, image processing, computer vision, machine learning, deep learning etc. In principle, all the complex calculations could be done using CPUs but for certain applications, the other processors make it more energy-efficient and process the heavy calculations faster. There are a few reasons for the selection of FPGA as a processor above GPU for this application.

The first advantage for FPGA is software portability. Programs for GPU are written using high-level languages and so it is faster and easier to debug codes. The FPGA can be programmed using hardware description languages such as VHDL or Verilog which take longer to debug, thereby increasing the development time. But, when there is a need to switch or upgrade to a hardware of the latest generation, the process of transferring the program in case of GPU might be challenging if the program is written considering certain architectural specification. In the case of FPGAs, the transfer 
of codes among Xilinx FPGA and Altera FPGA is not straightforward. But transferring codes from low to highperformance FPGA of the same family of Xilinx or Altera FPGA is comparatively simpler. Moreover, once the design is ready with an efficient program, the design can be used to design ASICs (Application Specific Integrated Circuits). The new generation Hardware description languages such as Chisel and Bluespec System Verilog(BSV) have also emerged to reduce the development time in FPGA.

Hardware advantage is the second attractive aspect of FPGA. FPGA are independent and can perform without the need of a CPU whereas GPUs can only be used as co-processors which means it is not PC-independent. Hence, it can be said that the FPGAs are more portable when it comes to the size of the hardware required for a similar task. GPUs were designed to achieve high operational parallelism but when there is no requirement for high parallel computations, a significant drop in the performance of the GPU is observed. On the other hand, FPGAs provide high flexibility which improves performance significantly in case of lesser data. The flow of data in an FPGA is from the input, through the designed circuit to the output. In the GPU, the flow of data happens back and forth between the CPU and the GPU due to which there is a higher consumption of energy. Therefore, FPGA turns out to be a better hardware choice which can be incorporated with the engine of the aircraft as it is both portable and energy-efficient (HajiRassouliha,, Taberner, Nash and Nielsen, 2018).

Health monitoring of aircraft can also be done using emerging technologies such as neural networks as discussed by Volponi, DePold, Ganguli and Daguang, (2000). Here they designed a health monitoring model using a neural network taking inputs from all the different modules of the gas turbine. For implementing such neural network-based models on the hardware it would become imperative to look into the advancements in GPUs and FPGAs which take place in the near future. GPUs are naturally better for implementation of emerging machine learning and deep learning algorithms. For example, the convolutional neural network models which involve calculations with matrices and GPU were made for such parallel matrix computation to enhance graphics (Krizhevsky, Sutskever and Hinton, 2012). However, the advancements in FPGA technology with the inclusion of CPUs also known as SoC (System on chip) has made them a formidable competitor of GPUs in this domain as well. FPGA-CPU SoCs were used to design their cloudscale which is indicative of the future possibilities and trends (Caulfield, Chung, Putnam, Angepat, Fowers, Haselman, Heil, Humphrey, Kaur, Kim, Lo, Massengill, Ovtcharov, Papamichael, Woods, Lanka, Chiou and Burger, 2016).

\section{BACKGROUND ON FILTERS}

\subsection{Averaging Filter}

An average filter, also known as the mean filter, belongs to the category of linear filters. The primary action performed by this one-dimensional windowed filter is smoothing of a signal. Given a filter of length N, it traverses across the signal and computes the average of the data points that is contained within the window length, according to the formula:

$$
Y=\sum_{i=1}^{N} \frac{x_{i}}{N}
$$

where $\mathrm{x}_{\mathrm{i}}$ represents the $\mathrm{i}^{\text {th }}$ data point, and $\mathrm{Y}$ is the output. One of the drawbacks of the use of averaging filter for noise removal is that it smoothens out the steep changes in the signal which can precede a fault event, and does not provide good results in the removal of outliers. However, the moving average filter remains popular in industry. A low window length filter keeps unwanted smoothing low. In this paper, we have implemented a 4 point averaging filter on both the Xilinx and Intel platforms.

\subsection{Median Filter}

The median filter belongs to the class of non-linear filters, wherein the window length, N, may contain even or odd sample values. In the case where $\mathrm{N}$ is odd, the sample values within the window is arranged in ascending order, and the middle value of the window is the output of the filter. In the case of $\mathrm{N}$ is even, the average of N/2 and N/2+1 is taken as the output. Thus, an element of averaging is introduced in the even length median filter. While an odd size median filter must return one of the input data points as the output, the even length filter typically returns a different value. An even length median filter can be considered as a hybrid of a median and mean filter. A median filter can be formulated as:

$$
Y=\operatorname{median}\left(x_{k-n}, x_{k-n+1}, \ldots, x_{k+n}\right)
$$

Here $x_{k}$ represents the data points. The median filter has been found to be very efficient in the removal of non-Gaussian noise. Unlike the averaging filter, the median does not smooth out sharp changes in the trend of data samples and is suitable for detecting outliers in the gas turbine signals. In the case of data with Gaussian noise, however, it takes numerous iterations for the filter output to converge to an optimum value. Our design comprises of a median filter with $\mathrm{N}=5$. The data point at $\mathrm{N}=3$, after sorting, is taken as the median in a given window.

\subsection{Recursive Median Filter}

The recursive median filter, or the RM filter, advances the simple median (SM) filter. For the purpose of calculating the 
output, the RM filter considers data outputs from previous iterations as well. It can be mathematically expressed as:

$$
Y=\operatorname{median}\left(y_{k-n}, y_{k-n+1}, \ldots, x_{k}, \ldots, x_{k+n}\right)
$$

Here $y_{k}$ is the output of the preceding iteration and $x k$ are the current data points. The recursive median filter is known to provide much better performance than the median filter in the removal of outliers in the fault signal. The blurring caused in the output signal when an RM filter is passed over it, remains one of its main disadvantages. In this paper, an RM filter with a window length of $\mathrm{N}=5$ is implemented.

\subsection{Weighted Recursive Median Filter}

The primary difference between the Weighted Recursive Median filter (WRM filter) and the Recursive Median Filter (RM filter) is that the data samples used in the RM filter are assigned certain pre-determined weight values to obtain the output of the WRM filter. These weights are assigned depending on the application. The WRM filter overcomes the disadvantages that are noted in RM filters such as blurring and produces better performance in terms of the rate at which the output is obtained (Verma and Ganguli, 2005; Payuna and Ganguli, 2010). The WRM filter can be expressed as:

$$
\begin{aligned}
& Y=\text { median }\left(m_{k-n} * y_{k-n}, m_{k-n+1} * y_{k-n+1}\right. \\
& \left.\ldots m_{k} * x_{k}, \ldots, m_{k+n} * x_{k+n}\right)
\end{aligned}
$$

Where $y_{k}$ are the output of preceding iterations, $x_{k}$ are the current data points and $m_{k}$ are the weights associated with them. The optimal weights chosen for obtaining the best filtered output was determined using the algorithm that tackles the optimization problem given as (Payuna and Ganguli, 2010):

$$
\begin{aligned}
& F\left(w_{-2}, w_{-1}, w_{0}, w_{1}, w_{2}\right) \\
& =\frac{1}{M} \sum_{i=1}^{M} \frac{1}{N} \sum_{j=1}^{N}\left|\widehat{z}_{j}-z_{j}{ }^{o}\right|
\end{aligned}
$$

\section{TEST SIGNALS}

A typical basic jet engine essentially has sensors for low rotor speed depicted as N1, high rotor speed as N2, fuel flow measurement as WF, and the exhaust gas temperature as EGT as shown in Fig 1. While modern engines have more sensors for pressure and temperature measurements, these four sensors are ubiquitous in operational jet engines, including older models. The data values provided by these sensors are utilized for determining the conditions of five different modules of the jet engine namely: fan denoted as FAN, lowpressure compressor as LPC, high-pressure compressor as
HPC, the low-pressure turbine and the high-pressure turbines as LPT and HPT respectively. The data generated from the sensors help in the detection of any deviations in the behavior of the modules compared to the expected behavior. The deviations in the modules are realized by observing the deviations in the sensor values represented as $\Delta \mathrm{N} 1, \Delta \mathrm{N} 2$, $\triangle \mathrm{WF}$ and $\triangle \mathrm{EGF}$ (Turner and Bajwa,1999).

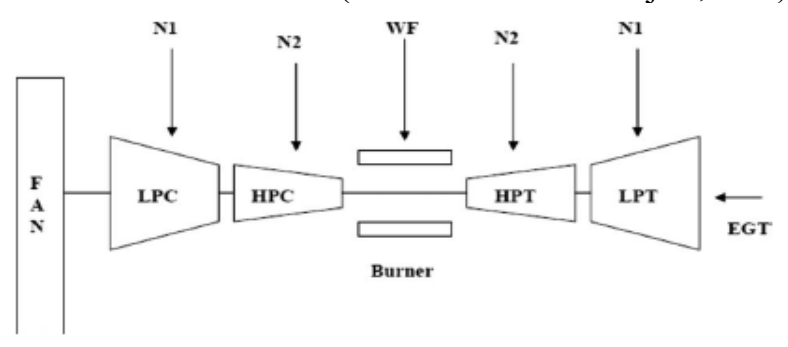

Fig 1: Representation of Basic Measurements in Gas

\section{Turbine}

The output signals of any sensor are not ideal signals that can be analyzed or used directly. The presence of noise in any output signal from the sensor is inevitable. But the presence of noise is not supposed to govern the judgement made on the condition and functioning of the engine. In order to detect these gas turbine faults, the signal needs to be pre-processed. For this, the performance of the filter needs to be verified. To represent this in mathematical form, the signals with noise can be described using the equations below:

$$
X=X^{\mathrm{o}}+\alpha
$$

Where the $\mathrm{X}^{\mathrm{o}}$ represents the ideal signal, i.e. the signal without noise, $\alpha$ represents the noise component, which is the reason for the deviation or the deltas in the signals. $\mathrm{X}$ is the complete output signal from the sensor. For the purpose of decision making and analysis it is necessary to have a signal with noise component being as small as possible else it could give erroneous prognostic results. Therefore, for noise reduction the sensors' output is to be passed through a function. Let that function be represented by $F($.). This function could be any filter, linear or non-linear, operating on the sensor output signal $\mathrm{X}$. The filter is given as:

$$
\widehat{X}=F(X)=F\left(X^{o}+\alpha\right)
$$

Where $\widehat{X}$ is the signal obtained after filtering.

For better understanding and visualization, in this paper three signals are considered:

\section{Step signal \\ 2. Ramp signal \\ 3. Combination of step and ramp signal}

The unit step response in Fig 2 is considered as it mimics abrupt faults. The ramp is considered to represent the gradual 
faults as shown in Fig 3. Fig 4 represents a combination of both the step signal and the ramp signal, which demonstrates an abrupt fault followed by a gradual fault. These signals are considered as the jet engine is susceptible to these three kinds of faults in real world scenarios. To make these signals more practical, the ideal signal is generated and white Gaussian noise is added to it. The standard deviation of the Gaussian noise generated is $\sigma=3.6262, \sigma=3.0541$ and $\sigma=3.2210$ for step signal, ramp signal and combination signal respectively. The signal thus generated occurs between $0 \mathrm{sec} \leq \mathrm{t} \leq 50 \mathrm{sec}$, with 250 data points. The signals simulated on MATLAB were sampled at every $0.2 \mathrm{sec}$. The resulting 250 samples were fed into the VERILOG simulation at $5 \mathrm{nsec}$ interval, and the obtained outputs were plotted against the original samples, on MATLAB. As the signal along with the noise after sampling has values with non-integer decimal precision as well, so for the purpose of simplicity in implementation on FPGA, the sampled points with decimal values are approximated to their nearest integer. In a window length of $\mathrm{N}=5$, each point being $4 \mathrm{bit}$ in length, the maximum height of the signal (along with the noise) needs to be below the magnitude of 15 considering only integer values. With a similar approach, these points can have more bits which will, in turn, increase the magnitude that can be considered for a signal. For example, considering 5-bit numbers will increase the magnitude limit to 32 . In case of requirement of better precision, the method of mapping can be used. Consider the situation where we have a 5-bit number, therefore, we can have 32 digital levels. Let us say we require a maximum magnitude of 8; then we can divide 8 into 32 levels, every level representing 0.25 . Similarly, this can be done for any number of bits.

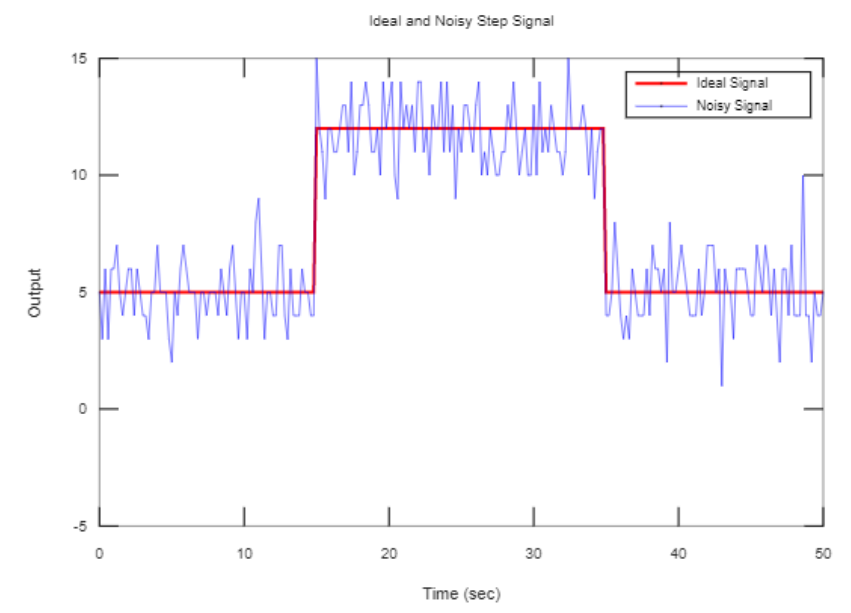

Fig 2: Step Signal Representing HPC Fault and its repair.

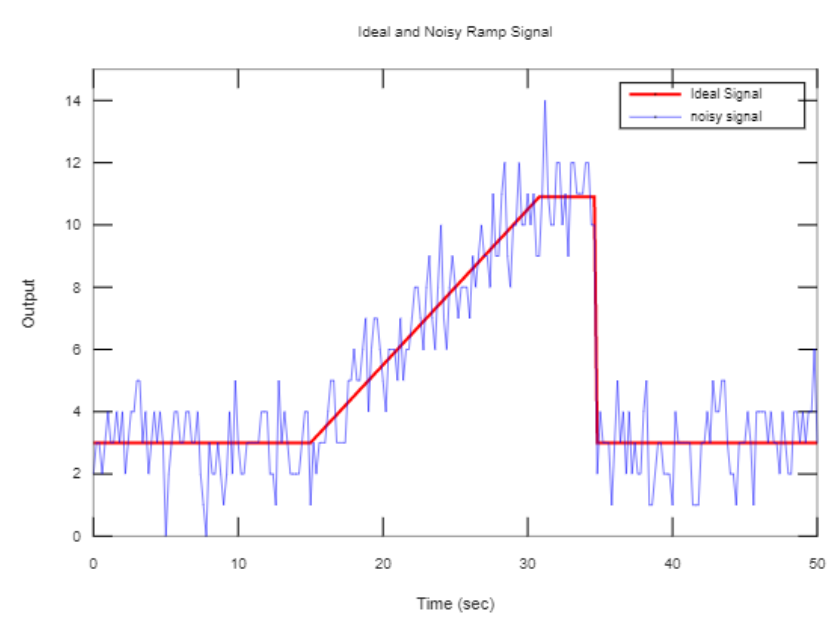

Fig 3: Ramp Signal Representing HPT Fault and its repair.

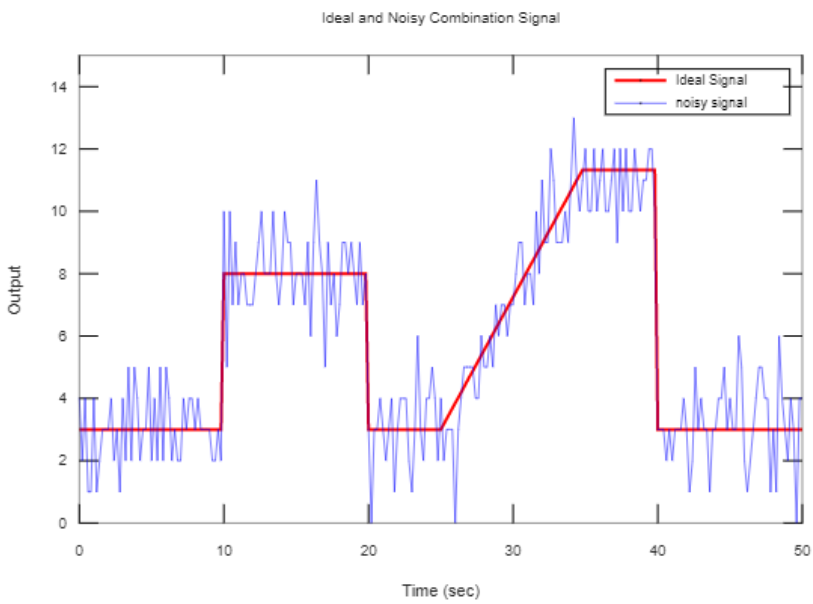

Fig 4: Combination Signal Representing a HPC fault and its repair followed by HPT fault and its repair 


\section{INTRODUCTION TO FPGAS}

A Field Programmable Gated Array - or FPGA, is an integrated circuit that possesses the capability of performing digital logic and application specific computations that can be customized by the end users depending on their need. Since its functionality is defined after it has been manufactured, therefore the term - "Field Programmable". FPGA's were originally brought into use in the 1980s as an alternative to PROMS (Programmable Read Only Memory) and PLDs (Programmable Logic Devices), because these were hard-wired, meaning that they could not be reprogrammed. With the advent of reprogrammable end-user chip technology, the growth of FPGA usage in the consumer market has seen an enormous increase over the past years, making its way into a variety of industrial domains such as automation, computation, networking and communication etc. Major companies are bringing about advanced technology in the form of high speed processors, dedicated computation blocks, large memory blocks in their FPGA devices.

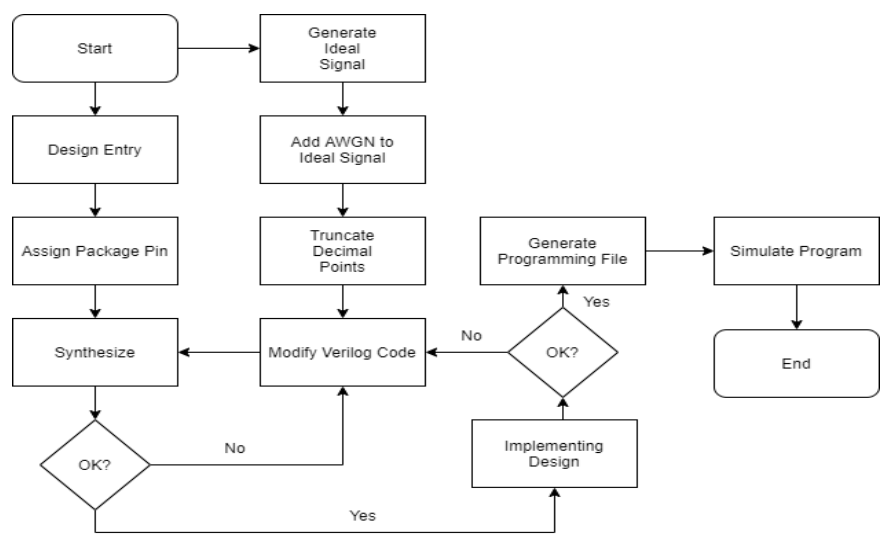

Fig 5: The process of generation of data

An FPGA is a semiconductor chip, consisting of millions of logic cells that can be programmed to perform any logic function. They are used to obtain the same level of performance, but at a visibly lower cost than its other alternatives such as that of a customized one-time programmable ASIC. Its structure consists of fundamental elements, which are enumerated next.

1. Input-Output Blocks (I/O Blocks): The logic gates that are present in the FPGA require inputs on which it operates, thereby giving the output for which it has been designed. For this purpose, FPGAs contains a large number of I/O pins and GPIOs (General Purpose Input Outputs) such as switches, push buttons etc. These direct the electrical signals into the logic circuitry and drive the output ports such as LEDs, displays etc.
2. Look-up Tables (LUTs): The smallest block in an FPGA is a Look-Up Table, which performs logic operations on the input received. Essentially, it is similar to a truth table, which holds values of the output that is desired for input combinations. In general, an $\mathrm{N}$ input LUT accesses $2 \mathrm{~N}$ memory locations. It will contain $\left(M=2^{\wedge} \mathrm{N}\right)$ configuration bits, and is capable of implementing $2^{\wedge} \mathrm{M}$ functions.

3. Flip Flops: Flip Flops are used as memory elements in FPGAs, wherein the output computed by the logic circuit is stored. It holds onto the value stored, till a new value is fed into it.

Xilinx manufactures commercially available reprogrammable FPGAs, whose basic architecture is arraybased. An array based structure implies that the logic blocks that make up a chip, is in the form of a 2-D matrix, and are linked to each other through both vertical and horizontal interconnects. The interconnect present in the chips are reprogrammable. These logic blocks are known as CLBs (Configurable Logic Blocks) in Xilinx, and are made up of Look-Up Tables.

In the FPGAs manufactured by INTEL, the basic logic block, is known as a Logic Element (LE), similar to a CLB present in a chip in the Xilinx FPGAs. Each of these logic elements is made up of one LUT, which can take up to four inputs to perform logic operations. For the output, one single flip flop is present in each LE.

The overall process of generating data and simultaneously programming the FPGA is shown in Fig 5.

\section{FPGA IMPLEMENTATION}

\subsection{Intel Quartus Development Suite}

The family of FPGAs offered by Intel (previously, Altera) contain dedicated and specialized high-performance DSP blocks, logic modules for logic implementation and large block memory on the system chip.

The design and synthesis for the purpose of the filter implementation was carried out on the Quartus Prime Lite Edition 19.1 on Windows. For the current problem, Cyclone $\mathrm{V}$ was chosen for the implementation. Modelsim-Intel FPGA Edition was used for simulation and optimization of the Verilog HDL program. The results of the filter design parameters when synthesized on the software are tabulated in Table 1.

The Weighted Recursive Median consumes the most logic modules in the design, while the simple median filter uses the least. The weights considered for the WRM filter are $[4,1,3,2,4],[4,1,2,2,3]$ and [2,2,2,1,3] for Step, Ramp and combinational signals respectively. The WRM filter also exhibits the maximum average fan-out, which indicates that it its power usage remains the highest amongst all. We deduce 
that the design of the median filter is the most efficient in terms of design and logic unit utilization on the chip.

\begin{tabular}{|c|c|c|c|c|}
\hline $\begin{array}{c}\text { Filters/ } \\
\text { Parameter } \\
\mathrm{s}\end{array}$ & $\begin{array}{c}\text { Logic } \\
\text { Utilizatio } \\
\mathrm{n} \text { in } \\
\text { ALMs } \\
(56,480)\end{array}$ & $\begin{array}{c}\text { I/O } \\
\text { Pins } \\
(480 \\
)\end{array}$ & $\begin{array}{c}\text { Averag } \\
\text { e Fan } \\
\text { Out }\end{array}$ & $\begin{array}{c}\text { Combinatio } \\
\text { n ALUT } \\
\text { Usage }\end{array}$ \\
\hline Average & 13 & 45 & 1.45 & 20 \\
\hline Median & 1 & 35 & 0.50 & 0 \\
\hline $\begin{array}{c}\text { Recursive } \\
\text { Median }\end{array}$ & 96 & 48 & 2.74 & 190 \\
\hline $\begin{array}{c}\text { Weighted } \\
\text { Recursive } \\
\text { Median }\end{array}$ & 154 & 28 & 3.56 & 291 \\
\hline
\end{tabular}

Table 1: Compilation and Synthesis Results of Quartus Development Suite

\subsection{Xilinx Vivado Development Suite}

Xilinx is a company that provides FPGAs to meet various requirements, such as greater performance, high end complicated operations at affordable costs. There are primarily five families of the FPGAs, namely VIRTEX, KINTEX, ARTIX, ZYNQ and SPARTAN, all of which are designed to meet the needs demanded by field-specific applications. There are certain FPGAs with on-chip processor features, known as System-on-Chips, for making the processing quicker and providing advanced capabilities to the devices.

For the implementation of the filters, the Xilinx board Nexys4 DDR which belongs to the ARTIX-7 low voltage family is used. The Nexys4 DDR kit has 15,850 logic slices, each with four 6-input LUTs and 8 flip-flops. The software used for the hardware description is the VIVADO Design Suite 2019.2. This software enables us to design and simulate the coded Verilog HDL program. Using the RTL analysis, the schematic based on the code can be obtained. At the end of the RTL analysis, the peak memory requirement is known. The VIVADO suite also facilitates synthesising the Verilog code which is specific to the board in use. After the synthesis, the board specific schematic is attained that has the descriptions of the number of input output ports, flip-flops, LUTs and DSP blocks used. Following the implementation of the filters, the descriptions shown in the software are tabulated in Table 2.

From the values in Table 2, it can be observed that the average filter's implementation uses the highest number of
I/O ports. The Recursive Median Filter needs the highest peak memory. The Weighted Recursive Median filter implementation uses the highest flip-flops and LUT. The weights considered for the WRM filter are $[4,1,3,2,4]$, $[4,1,2,2,3]$ and $[2,2,2,1,3]$ for Step, Ramp and combinational signals respectively. These values might vary with the FPGA board and the algorithms used for implementation. For Median, Recursive Median and Weighted Recursive Median filters the sorting method used here is bubble sort. Usage of some other sorting algorithm will yield different results.

\begin{tabular}{|c|c|c|c|c|}
\hline Filters/ & $\begin{array}{c}\text { Peak } \\
\text { Pemory } \\
(\mathrm{MB})\end{array}$ & $\begin{array}{c}\text { I/O } \\
\text { Ports }\end{array}$ & $\begin{array}{c}\text { Flip } \\
\text { Flops }\end{array}$ & $\begin{array}{c}\text { LUT } \\
\text { Usage }\end{array}$ \\
\hline Average & 1414.855 & 68 & 0 & 1 \\
\hline Median & 1481.805 & 28 & 0 & 0 \\
\hline $\begin{array}{c}\text { Recursive } \\
\text { Median }\end{array}$ & 2022.215 & 28 & 0 & 0 \\
\hline $\begin{array}{c}\text { Weighted } \\
\text { Recursive } \\
\text { Median }\end{array}$ & 1649.820 & 12 & 3 & 113 \\
\hline
\end{tabular}

Table 2: Compilation and Synthesis Results of Vivado Development Suite

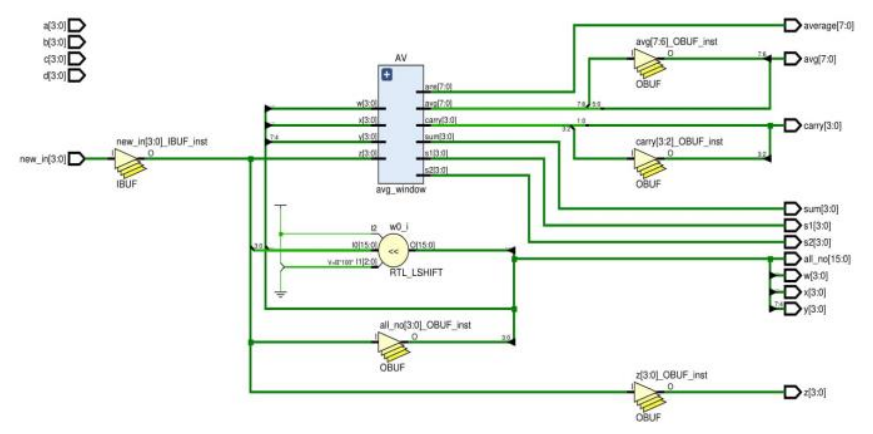

Fig 6: RTL Schematic of Averaging filter

The data is generated through MATLAB and decimal truncated, following which it is fed into the VERILOG code. The code is repeatedly modified till a synthesizable design is obtained. Fig 6 and Fig 7 depicts the RTL synthesis diagram obtained of the average and recursive median filter respectively, after compilation of the Verilog program. 


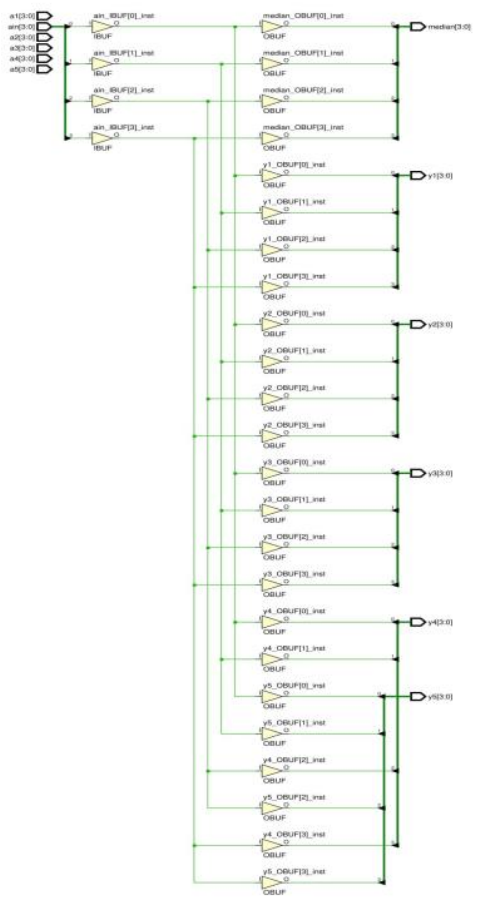

Fig 7: RTL Schematic of Recursive Median Filter.

\section{PERFORMANCE ANALYSIS OF FILTERS}

The noise can get added to the signal during the creation or even during the transmission of data. It is also possible that the noise at times overpowers the signal itself. This becomes a concern as the signal is completely corrupted and retrieval becomes a difficult challenge. Various methodologies are used for signal cleaning and noise removal. The next hurdle is to identify the best possible solution for the removal of noise from the signal. The extent of denoising is compared by certain parameters such as:

1. PSNR: peak signal to noise ratio; which is the ratio of the maximum possible power of the signal to that of the power of the noise that affects the signal's representation.

2. MSE: mean squared error; it is squared to remove the negative components of the noisy signal and is averaged out to give more weightage to the larger deviations. Lesser the value of MSE the better in the considered signal.

3. SNR: signal to noise ratio; it the comparison of the RMS value of the ideal or expected signal to that of the RMS of the noise content of the signal.

4. RMSE: Root mean squared error, it quantifies how far or close is the spread of noise from the original signal. (Hore and Ziou, 2010; Saladi and Prabha, 2017)

The motive of considering all these parameters for comparison is to obtain a clear demarcation between the signals which are compared. In this paper, the outputs of the filters are compared using the SNR parameter. Any ratio more than 1:1 indicates that the signal has more required component, than the noise component. The larger the value of the SNR the better is the obtained output. This can be represented by the equation:

$$
S N R=20 * \log _{10} \frac{R M S \text { Signal }}{\text { RMS Noise }}
$$

A complete removal of noise is not possible with these filters, but any improvement in the signal by reducing the noise component can result in better performance. As per the tabulated values in Table 3, it can clearly be seen that the filter with the best performance is the Weighted Recursive Median filter followed by Recursive, Median and Average in the same order. The results obtained below are from the FPGA simulation. The input for the simulation was a noisy signal whose data points were rounded off to the nearest integer. For simplicity 4-bit numbers were taken which could give numbers between 0-15 only and the peak of the signal considered was 15 . Thereby leaving no scope for decimal values. Hence the outputs obtained were also integers. The results are based on the above considerations.

The increment in the window length of the filter results in the improvement, which is what essentially occurs in the Weighted Recursive Median Filter. It is necessary to select the correct set of weights for the WRM filter for better performance. Fig. 8-16 illustrates the plots of the obtained filtered output. On implementing the same filters on MATLAB with non-truncated input values and comparing the results, we note that the filter designs on FPGA perform with a relative error of $(4 \%-15 \%)$.

\begin{tabular}{|c|c|c|c|}
\hline $\begin{array}{c}\text { Filters / } \\
\text { Signals }\end{array}$ & $\begin{array}{c}\text { Step } \\
(\sigma=3.6262)\end{array}$ & $\begin{array}{c}\text { Ramp } \\
(\sigma=3.0541)\end{array}$ & $\begin{array}{c}\text { Combination } \\
(\text { Step and } \\
\text { Ramp }) \\
(\sigma=3.2210)\end{array}$ \\
\hline Average & 8.130 & 9.841 & 10.391 \\
\hline Median & 16.552 & 13.500 & 12.599 \\
\hline $\begin{array}{c}\text { Recursive } \\
\text { Median }\end{array}$ & 16.640 & 16.248 & 13.248 \\
\hline $\begin{array}{c}\text { Weighted } \\
\text { Recursive } \\
\text { Median } \\
\text { (Weights })\end{array}$ & 17.068 & 16.560 & 13.471 \\
$(4,1,3,2,4)$ & $(4,1,2,2,3)$ & $(2,2,2,1,3)$ \\
\hline
\end{tabular}


Table 3: SNR of Tested Signals

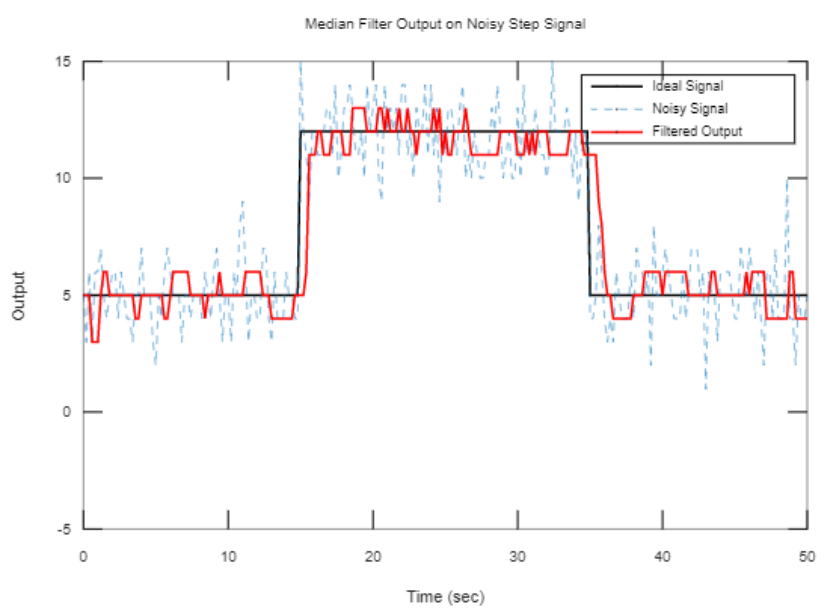

Fig 8: Median Filter Output on Noisy Step Signal

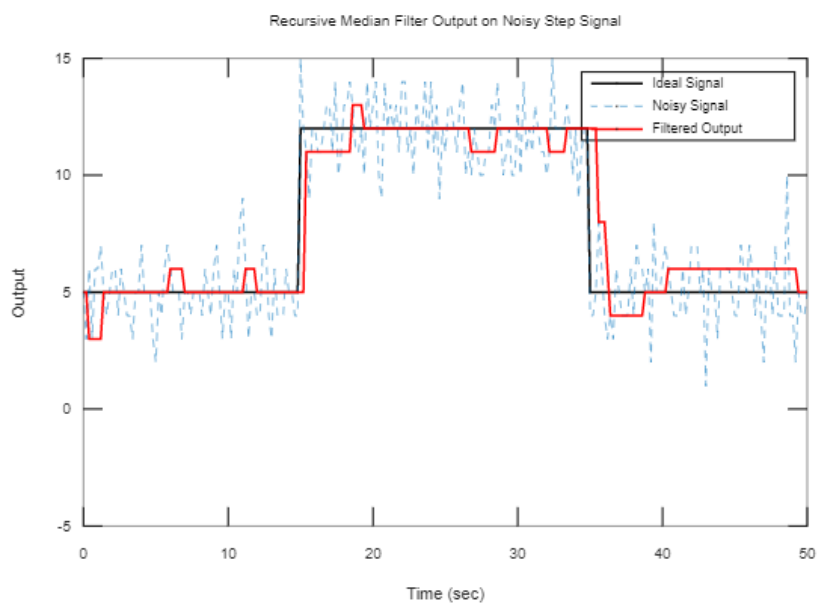

Fig 9: Recursive Median Filter Output on Noisy Step Signal

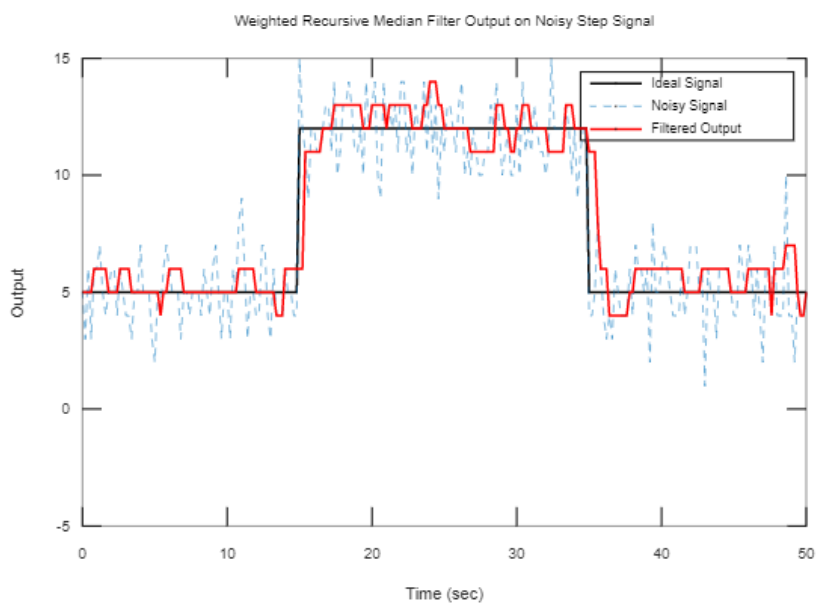

Fig 10: Weighted Recursive Median Filter Output on Noisy Step Signal

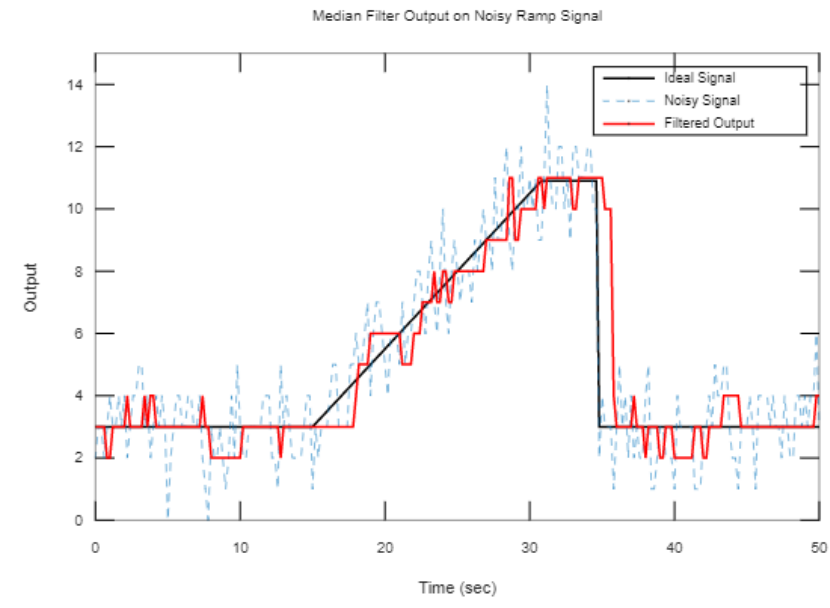

Fig 11: Median Filter Output on Noisy Ramp Signal

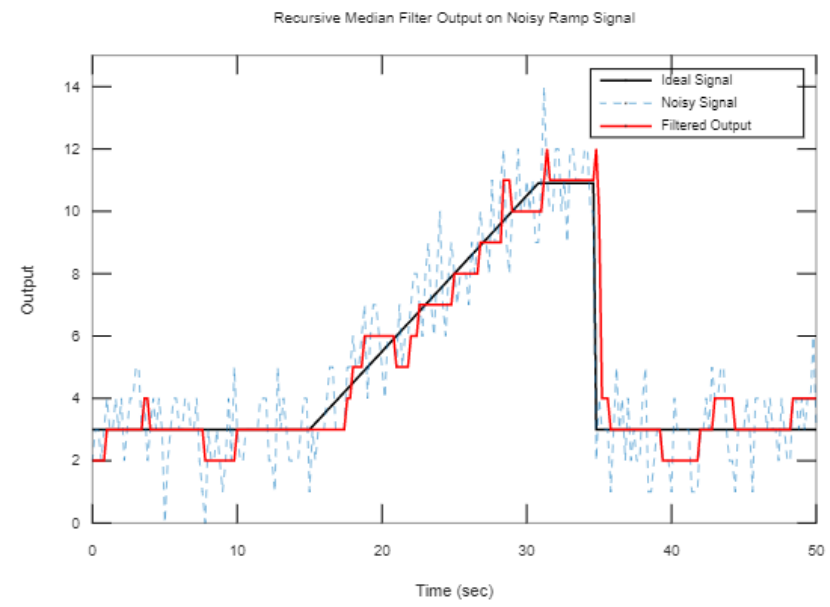

Fig 12: Recursive Median Filter Output on Noisy Ramp Signal

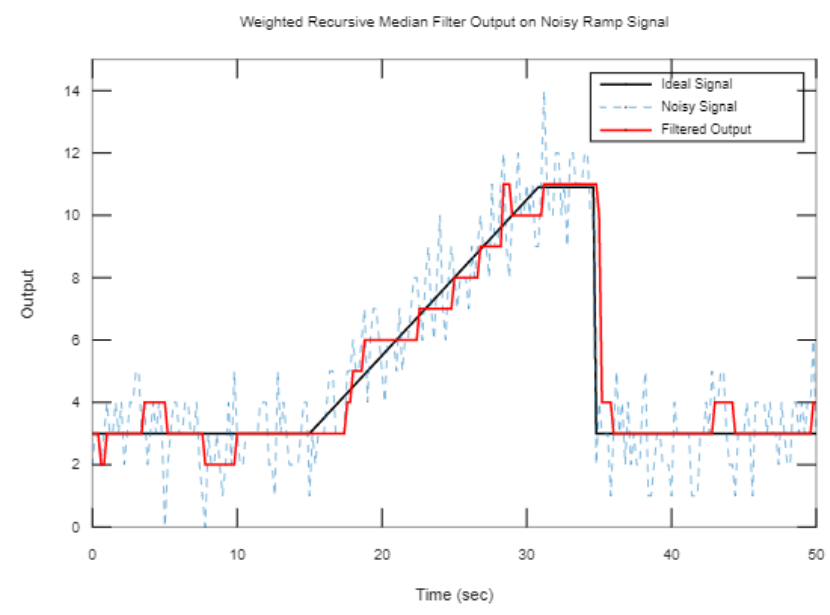

Fig 13: Weighted Recursive Median Filter Output on Noisy Ramp Signal 


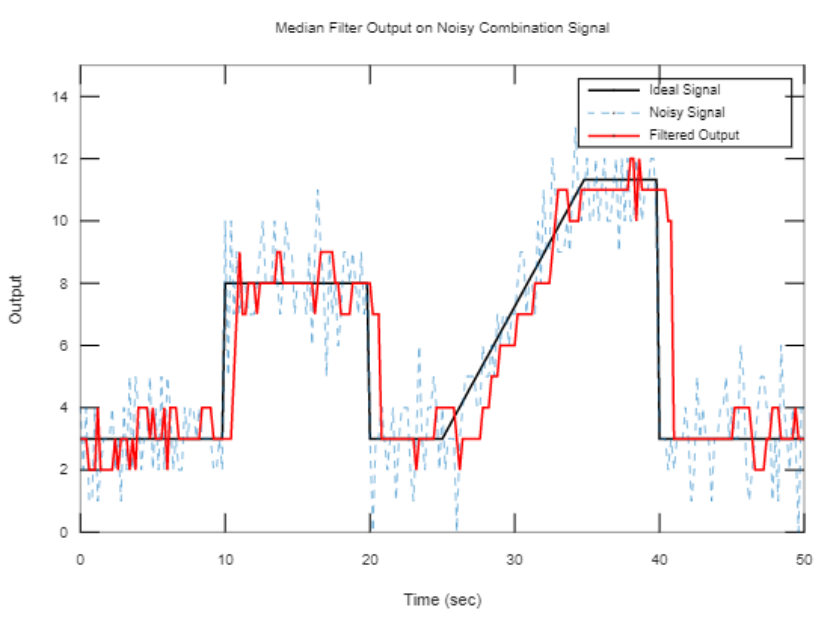

Fig 14: Median Filter Output on Noisy Combination Signal

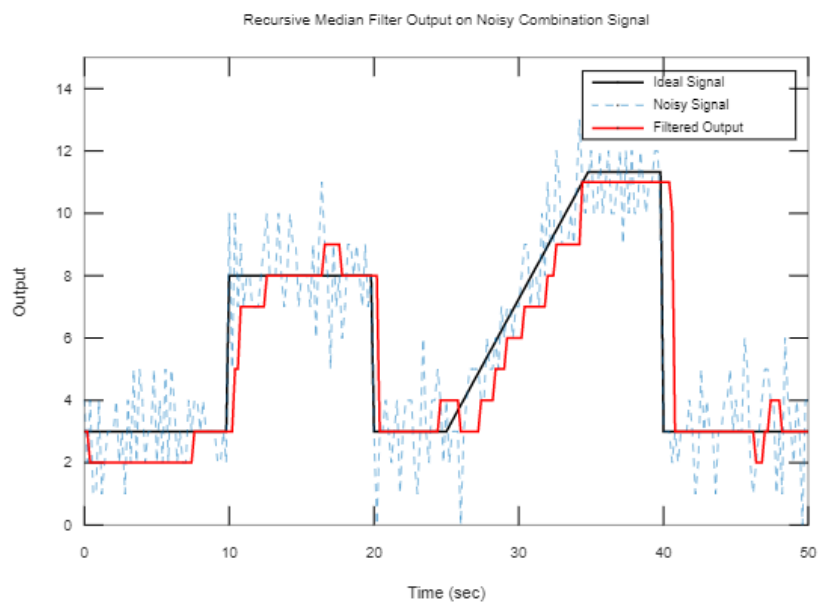

Fig 15: Recursive Median Filter Output on Noisy Combination Signal

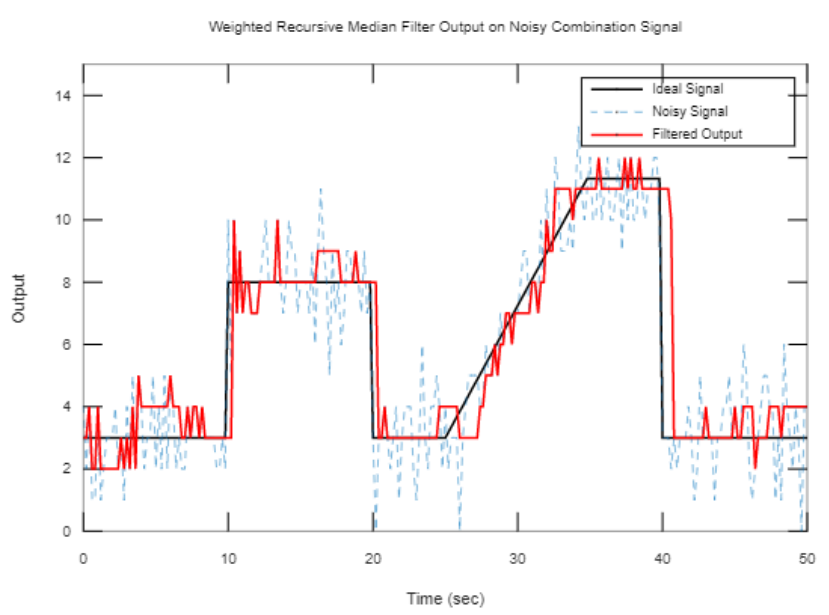

Fig 16: Weighted Recursive Median Filter Output on Noisy Combination Signal

\section{DISCUSSION}

Based on the numerical analysis performed, we deduce that the weighted recursive median filter provides the most effective results in the removal of noise from data. The averaging filter performs poorly and succeeds in removing nominal noise from the faulty signal generated. This is primarily due to the fact that the averaging filter causes smoothening of the data points near sharp changes in the measurement signal which leads to loss of vital attributes contained in the signal that can prove to be essential to the task of the removal and isolation of faults from the noisy signal as suggested by Payuna and Ganguli, (2010). The average filter also fares poorly in the preservation of edges present in the test signal, thus causing performance degradation. Therefore, the averaging filter is not the ideal choice for noise removal and fault isolation.

The median filter, on the other hand performs well in the feature preservation for the faulty signals, unlike the linear averaging filter. This can be seen through the reported results which show significantly better performance metrics when tested on the error signals. Median filters are more sensitive to the existence of outliers in the data. Its variant, the recursive median filter achieves even better results. The outcome obtained depicts the superior performance in fault isolation, and therefore is better suited than the previously noted filters, as it takes into account the output values of previous iterations as well.

The weighted recursive median, which involves the assignment of weights to the recursive median filter exhibits the greatest efficiency in filtering out noise, as it allows the fine-tuning of the filter according to the signal under test.

It should be mentioned that the results in this paper use simulated data. Using simulated data allows easy comparison of the algorithms in terms of error norms as the ideal signal is known. However, our work can easily be used by practitioners working in companies who may have access to real world test data.

\section{CONCLUSION}

The performance of linear and non-linear filters are evaluated through their implementation on FPGAs for the purpose of improved prognostics of gas turbine engines, wherein the filters are employed to detect and isolate faults in the data signals. The signals modelled for this task include the step signal which represents an abrupt fault and a ramp signal which indicates a gradual fault occurring in the data received from measurements of the engine. Two different platforms, namely the Vivado Design Software Suite by Xilinx and the Quartus Pro Lite Edition by Intel are used for the design and simulation of the filters. The filters modelled are a five point averaging filter, which is a linear filter; a simple median filter, a recursive median filter, which comprise of window length of $\mathrm{N}=5$ and a weighted recursive median filter that are 
non-linear in nature. The architectural difference between the two designing platforms is noted. Finally, the performance analysis of the filters was conducted based on the signal-tonoise ratio obtained, by recording the difference in the SNR of the noisy data and subsequently, the data received after filtering. It was hence, shown that the weighted recursive median filter provides superior results than the other filters designed for testing. In subsequent research, the real-time implementation of the proposed filters can be carried out on FPGA to highlight the performance benefits offered by the devices such as high sampling rate for input data, input/output rates, etc.

The FPGA implementation of the denoising filters is likely to spread this technology in the prognostics community and lead to considerable benefits in terms of real time implementation.

\section{REFERENCES}

Volponi, A. (2014) Gas turbine engine health management: Past, present, and future trends. J. Eng. Gas Turbines Power 2014, 136. doi: 10.1115/1.4026126

Borguet S., Henriksson M., McKelvey T. \& Léonard O. (2011). A study on engine health monitoring in the frequency domain, J. Eng. Gas Turbines Power., Vol. 133 Issue 8 doi: 10.1115/1.4002832

Zhao N., Wen, X., \& Li, S. (2016). A Review on gas turbine anomaly detection for implementing health management Volume 1: Aircraft Engine; Fans and Blowers; Marine. doi: 10.1115/GT2016-58135

Fentaye A., Baheta A., Gilani S., \& Kyprianidis K. (2019). A review on gas turbine gas-path diagnostics: State-ofthe-art methods, challenges and opportunities Aerospace, 6(7), 83 doi: 10.3390/aerospace6070083

Ganguli R. (2012) Jet engine gas-path measurement filtering using center weighted idempotent median filters, Journal of Propulsion and Power Vol. 19 Issue 5. doi: $10.2514 / 2.6186$

Luppold R., Roman J., Gallops G., \& Kerr L. (1989). Estimating in-flight engine performance variations using kalman filter concepts. 25th Joint Propulsion Conference. doi: 10.2514/6.1989-2584

Pourbabaee B., Meskin N., \& Khorasani K. (2016). Sensor Fault Detection, Isolation, and Identification Using Multiple Model Based Hybrid Kalman Filter for Gas Turbine Engines. IEEE Transactions on Control Systems Technology, Vol. 24, Issue 4 doi: 10.1109/TCST.2015.2480003

Lu F., Huang Y., Huang J., \& Qiu X. (2018). A Hybrid Kalman Filtering Approach Based on Federated Framework for Gas Turbine Engine Health Monitoring IEEE Access: The Multidisciplinary
Open Access Journal, Vol 6. doi: 10.1109/ACCESS.2017.2780278

Lu F., Ju H., \& Huang J. (2016). An improved extended Kalman filter with inequality constraints for gas turbine engine health monitoring Aerospace Science and Technology, Vol. 58, 36-47. doi: 10.1016/j.ast.2016.08.008

Daroogheh N., Baniamerian A., Meskin N., \& Khorasani K. (2015) A hybrid prognosis and health monitoring strategy by integrating particle filters and neural networks for gas turbine engines IEEE Conference on Prognostics and Health Management(PHM) doi: 10.1109/ICPHM.2015.7245020

Liu, J., Liu, J., Yu, D., Kang, M., Yan, W., Wang, Z., \& Pecht, M. (2018) Fault detection for gas turbine hot components based on a convolutional neural network Energies, Vol 11 Issue 8. doi: 10.3390/en11082149

Tayarani-Bathaie, S. S., Vanini, Z. N. S., \& Khorasani K. (2012). Fault detection of gas turbine engines using dynamic neural networks. 2012 25th IEEE Canadian Conference on Electrical and Computer Engineering (CCECE) doi: 10.1109/CCECE.2012.6334837

Y. G. Li , Ghafir A., Wang L., Singh R., Huang K. \& Feng X.(2011). Nonlinear multiple points gas turbine offdesign performance adaptation using a genetic algorithm, J. Eng. Gas Turbines Power, Vol. 133 Issue 7. doi: 10.1115/1.4002620

Li, Y. G., Abdul Ghafir, M. F., Wang, L., Singh, R., Huang, K., Feng, X., \& Zhang, W. (2012). Improved multiple point nonlinear genetic algorithm based performance adaptation using least square method, J. Eng. Gas Turbines Power, Vol 134 Issue 3. doi: $10.1115 / 1.4004395$

Henaut J., Dragomirescu D., \& Plana R. (2009). FPGA based high date rate radio interfaces for aerospace wireless sensor systems 2009 Fourth International Conference on Systems doi: 10.1109/ICONS.2009.28

Fay D., Campbell S., Miller G., \& Connors D. (2007). Teaching fault tolerant FPGA design for aerospace applications. 2007 IEEE International Conference on Microelectronic Systems Education (MSE'07). doi: 10.1109/MSE.2007.81

Gankidi P. R., \& Thangavelautham J. (2017). FPGA architecture for deep learning and its application to planetary robotics. 2017 IEEE Aerospace Conference doi: 10.1109/AERO.2017.7943929

Verma R., \& Ganguli R. (2005). Denoising jet engine gas path measurements using nonlinear filters 
IEEE/ASME Transactions on Mechatronics, Vol. 10 Issue 4. doi: 10.1109/TMECH.2005.852454

Payuna U. \& Ganguli R., (2010) Jet engine health signal denoising using optimally weighted recursive median filters J. Eng. Gas Turb. Power vol. 132 Issue 4 doi: 10.1115/1.3200907

Hore A \& Ziou D. (2010) Image quality metrics: PSNR vs. SSIM. 20th International Conference on, pp. 23662369. IEEE Pattern Recognition (ICPR), 2010. doi: 10.1109/ICPR.2010.579

Saladi S., \& Prabha, N. (2017). Analysis of denoising filters on MRI brain images. International Journal of Imaging Systems and Technology, vol.27 Issue 3 doi: 10.1002/ima.22225

Turner K. \& Bajwa A. (1999) A survey of aircraft engine health monitoring systems AIAA/ ASME/ SAE/ ASEE Joint Propulsion Conference and Exhibit 2024 June 1999 doi: 10.2514/6.1999-2528

Fahmy S.A, Cheung P. \& Luk W. (2009) High-throughput one-dimensional median and weighted median filters on FPGA IET Computers \& Digital Techniques 2009 doi: 10.1049/iet-cdt.2008.0119

Benkrid K., Crookes D., \& Benkrid A. (n.d.). (2002) Design and implementation of a novel algorithm for general purpose median filtering on FPGAs 2002 IEEE International Symposium on Circuits and Systems. doi: 10.1109/ISCAS.2002.1010482

Hyeong-Seok Yu, Joon-Yeop Lee, \& Jun-Dong Cho (1999) A fast VLSI implementation of sorting algorithm for standard median filters Twelfth Annual IEEE International ASIC/SOC Conference doi: 10.1109/ASIC.1999.806540

HajiRassouliha, A., Taberner, A. J., Nash, M. P., \& Nielsen, P. M. F. (2018). Suitability of recent. hardware accelerators (DSPs, FPGAs, and GPUs) for computer vision and image processing algorithms. Signal Processing: Image Communication, 68, 101119. doi:10.1016/j.image.2018.07.007

Volponi, A. J., DePold, H., Ganguli, R., \& Daguang, C. (2000). The Use of Kalman Filter and Neural Network Methodologies in Gas Turbine Performance Diagnostics: A Comparative Study. Volume 4: Manufacturing Materials and Metallurgy; Ceramics; Structures and Dynamics; Controls, Diagnostics and Instrumentation; Education. doi:10.1115/2000-gt-0547

Krizhevsky A., Sutskever I., Hinton G.E. (2012) ImageNet Classification with deep convolutional neural networks, Adv. Neural Inf. Process. Syst., 2012, pp. $1-9$.

Caulfield A. M., Chung E.S, Putnam A., Angepat H., Fowers J., Haselman M., Heil S., Humphrey M., Kaur P., Kim J.Y, Lo D., Massengill T., Ovtcharov K., Papamichael M., Woods L., Lanka S., Chiou D. and Burger D. (2016) A cloud-scale acceleration architecture, 2016 49th Annual IEEE/ACM International Symposium on Microarchitecture (MICRO), Taipei, 2016, pp. 1-13, doi: 10.1109/MICRO.2016.7783710. 